\title{
Jitter Studies for the Secondary and Tertiary Mirror Systems on the Thirty Meter Telescope
}

\author{
Virginia Ford*a, Christopher Carter ${ }^{\mathrm{a}}$, Christophe Delrez ${ }^{\mathrm{b}}$, Pierluigi Fumi ${ }^{\mathrm{c}}$, Eric Gabriel ${ }^{\mathrm{b}}$, Daniele \\ Gallieni $^{\mathrm{c}}$ \\ ${ }^{a}$ Thirty Meter Telescope International Observatory Corporation, 1111 S. Arroyo Parkway, Pasadena, \\ CA, USA 91105 \\ ${ }^{\mathrm{b}}$ Advanced Mechanical \& Optical Systems S.A., Liege Science Park, Rue des Chasseurs Ardennais \\ 2, Angleur 4031, Belgium \\ ${ }^{c}$ A.D.S. International S.r.l., Via Roma, 87, 23868 Valmadrera (LC), Italy
}

\begin{abstract}
The Secondary Mirror System (M2S) and Tertiary Mirror System (M3S) of the Thirty Meter Telescope (TMT) consist of passively mounted mirrors supported in kinematic cell assemblies that are moved during telescope tracking to counteract effects of changing zenith angle and thermal gradients within the telescope structure. TMT is concerned that the requirements for pointing jitter during Adaptive Optics tracking for the M2 and M3 Systems are very challenging with a risk of requiring complex stabilization systems for compliance. Both systems were researched to determine whether similar un-stabilized hardware exists that can meet the TMT jitter requirements. Tests using representative TMT tracking motions were then performed to measure jitter on similar existing hardware. The results of these hardware tests have been analyzed. Test results, remaining risk assessment and further testing plans are presented.
\end{abstract}

Keywords: jitter, filtered jitter, tracking, hexapod, following error, rotation-tilt gimbal, alt-az telescope, mirror positioner

\section{INTRODUCTION}

\subsection{TMT telescope overview}

The TMT telescope is a Ritchey-Chrétien three mirror design as shown in Figure 1. The three mirror telescope rotates in azimuth and altitude to acquire and track astronomical objects. The TMT dome and telescope assembly is shown in Figure 2.

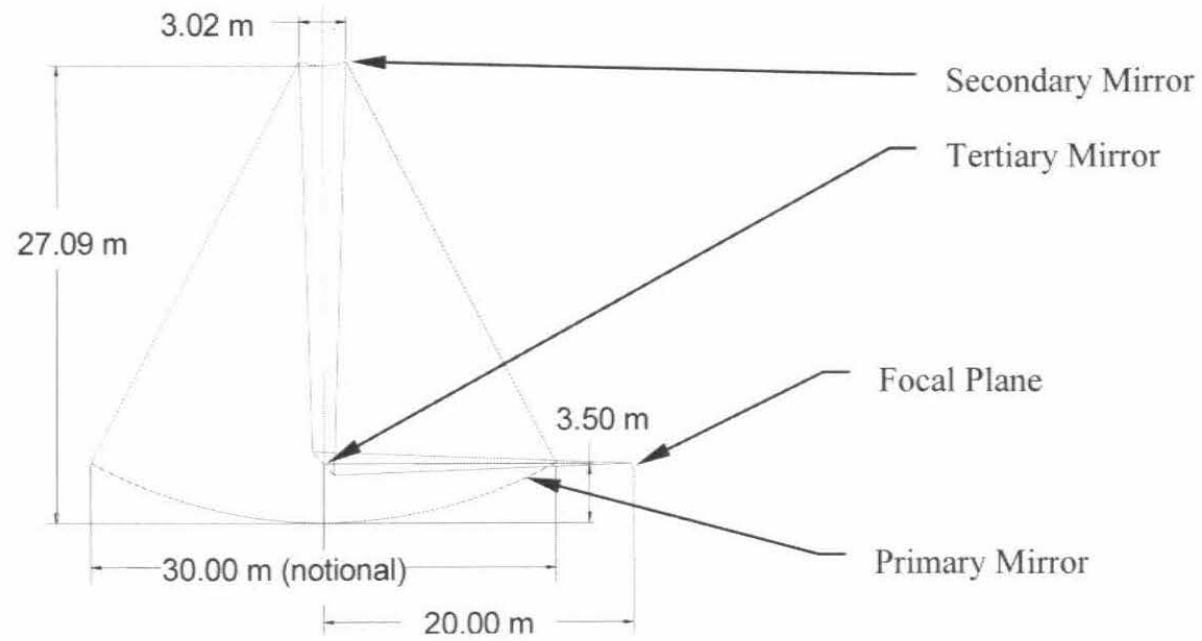

Figure 1. TMT Optical Design 


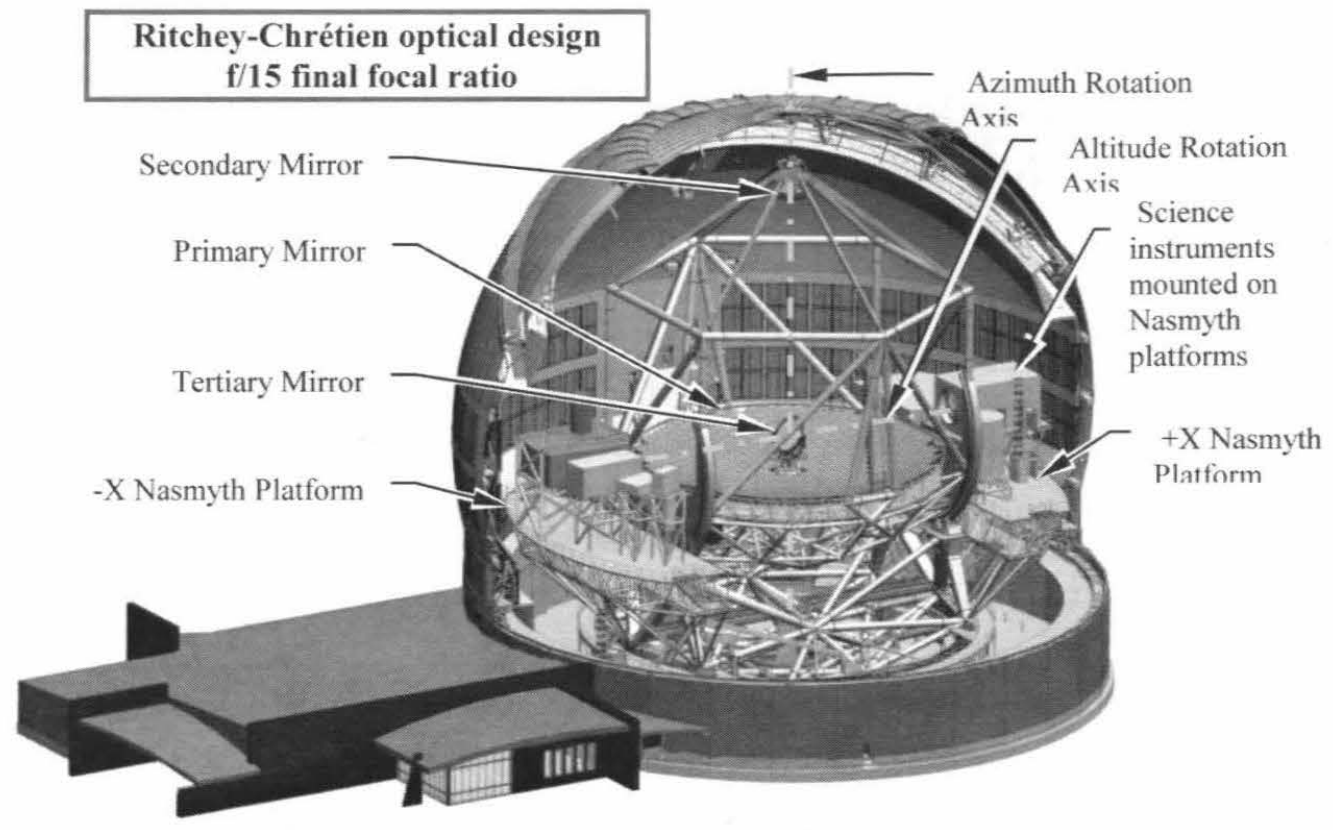

Figure 2. TMT Dome and Telescope Assembly

The primary mirror forms the aperture stop of the telescope. It consists of 492 hexagonally-shaped aspheric hyperboloid segments that are positioned and warped to form a co-phased 30 meter diameter $\mathrm{f} / 1$ mirror.

The secondary mirror is a passively-supported convex hyperboloid asphere, 3.1 meter diameter, with a radius of curvature of -6.2277 meters and a conic constant of -1.318 . It translates in $x, y$, and $z$ and rotates in $\mathrm{Rx}$ and Ry to correct telescope focus and coma.

The tertiary mirror is an elliptical flat mirror 2.5 by 3.5 meters in size. It rotates about the optical axis between the primary mirror and secondary mirror and tilts about an axis passing through the telescope elevation axis to direct the optical beam into the selected active instrument on the Nasmyth platforms.

The science instruments are located on two Nasmyth platforms on both sides of the elevation axis. The Nasmyth platforms rotate in azimuth, but not in altitude. Because of this, the tertiary mirror must change tilt and rotation to maintain pointing during tracking for all instruments that are not located on the elevation axis.

Atmospheric wavefront correction is performed by a facility instrument called Narrow Field Infra-Red Adaptive Optics System (NFIRAOS). NFIRAOS measures atmospheric effects using laser guide stars or natural guide stars. The telescope range of motion encompasses the full visible sphere of the night sky excluding the spherical zone that is 1 degree away from zenith and the spherical zone from 65 degrees zenith angle to 90 degrees zenith angle. Because of the range of motion, and the requirement for natural guide stars covering the entire range, available stars in some locations in the sky are not very bright. The brightness of natural guide stars influences TMT jitter requirements.

\subsection{Secondary mirror system deseription}

The secondary mirror system is shown in Figure 3 mounted onto the telescope top end structure. It consists of a cell assembly and a hexapod positioner. The cell assembly and the positioner will be developed with separate contracts by suppliers that will be selected by competitive bid during the secondary mirror construction phase.

The hexapod positioner is based on a Stewart platform with six actuated legs that coordinate to locate the secondary mirror as commanded. Encoders on each leg provide motion feedback. 


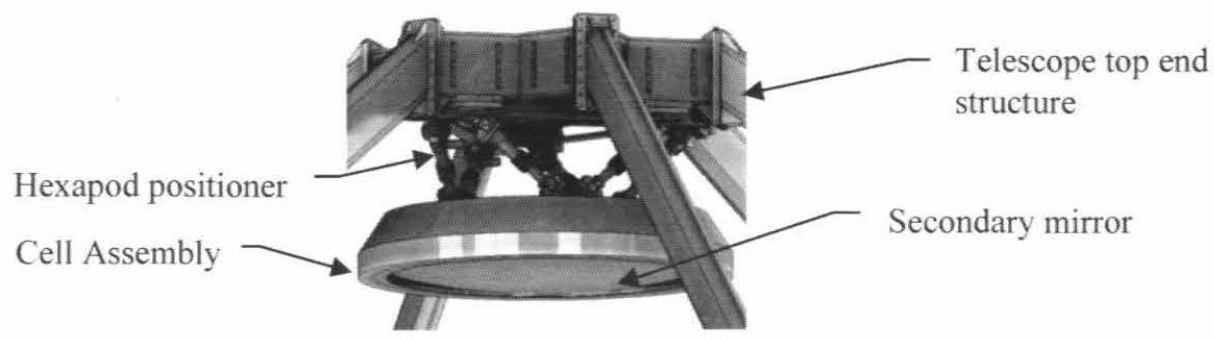

Figure 3. Secondary mirror system mounted to the telescope top end structure

\subsection{Tertiary mirror description}

The tertiary mirror system is shown in Figure 4 mounted onto the telescope M3 Tower structure. The tertiary mirror system consists of a cell assembly and an altitude-azimuth-style positioner that provides rigid-body rotation and tilt of the mirror cell assembly. The cell assembly and the positioner are being developed as a work share contribution by the Changchun Institute of Optics, Fine Mechanics and Physics, CAS, (CIOMP) in Changchun, China.

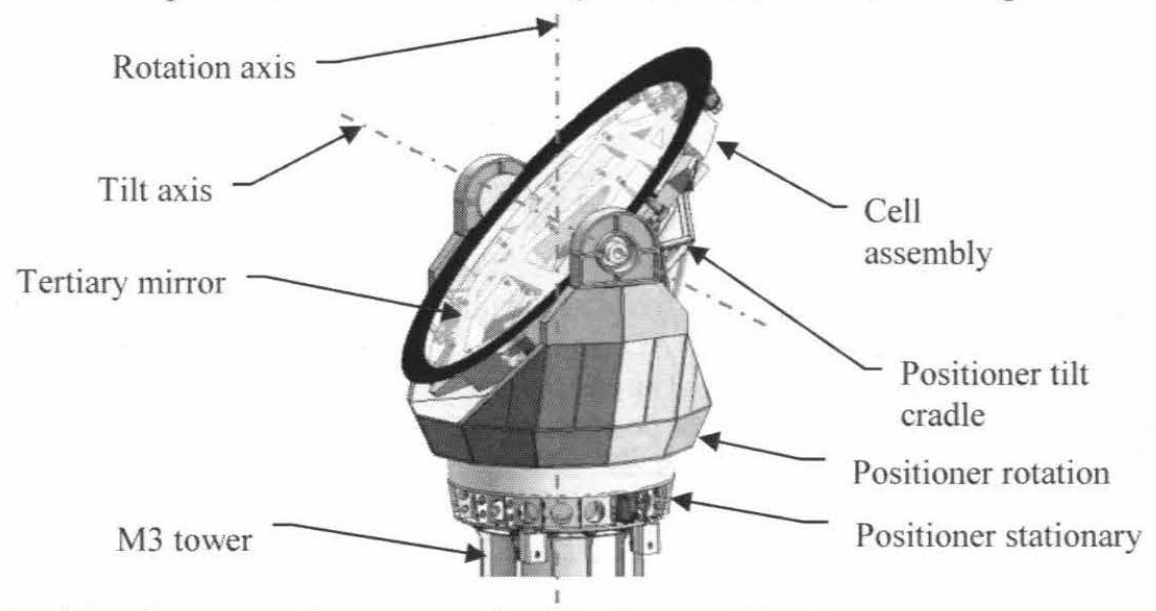

Figure 4. Tertiary mirror system shown mounted to the M3 tower of the telescope structure

\subsection{Jitter on the TMT telescope}

Jitter is defined for TMT as the change in image position during observing. The TMT error budget allocates image jitter error into the following sources: Control Noise; Wind Jitter Residual; and Vibration Jitter. Control Noise (CN) jitter is caused by all components in a motion drive system such as gears, bearing stiction, motor torque ripple, encoder resolution, cable wrap stiction and control loop noise. Wind Jitter Residual contains all image jitter motions due to wind buffeting. Vibration Jitter includes all image jitter motions caused by equipment induced vibrations and micro-seismic vibrations. The jitter of concern that will be discussed in this presentation is $\mathrm{CN}$ jitter. For the secondary and tertiary mirror systems, the motion of the positioners as they move the mirrors during observing causes $\mathrm{CN}$ jitter.

There are two types of TMT CN jitter requirements: error allocations for image jitter during guided-tracking observations and during observations using the adaptive optic system, NFIRAOS.

Guided-tracking image jitter is corrected during observing by the telescope control system. Image location is monitored during closed-loop operation and sent to the telescope control system which commands the telescope pointing subsystems to correct the pointing offset. The bandwidth for pointing correction is $0.1 \mathrm{~Hz}$ which filters out jitter occurring at frequencies lower than $0.1 \mathrm{~Hz}$. The guided-tracking correction filter can be represented as a high pass Butterworth filter with a $0.1 \mathrm{~Hz}$ break frequency as shown in Figure 5a. 

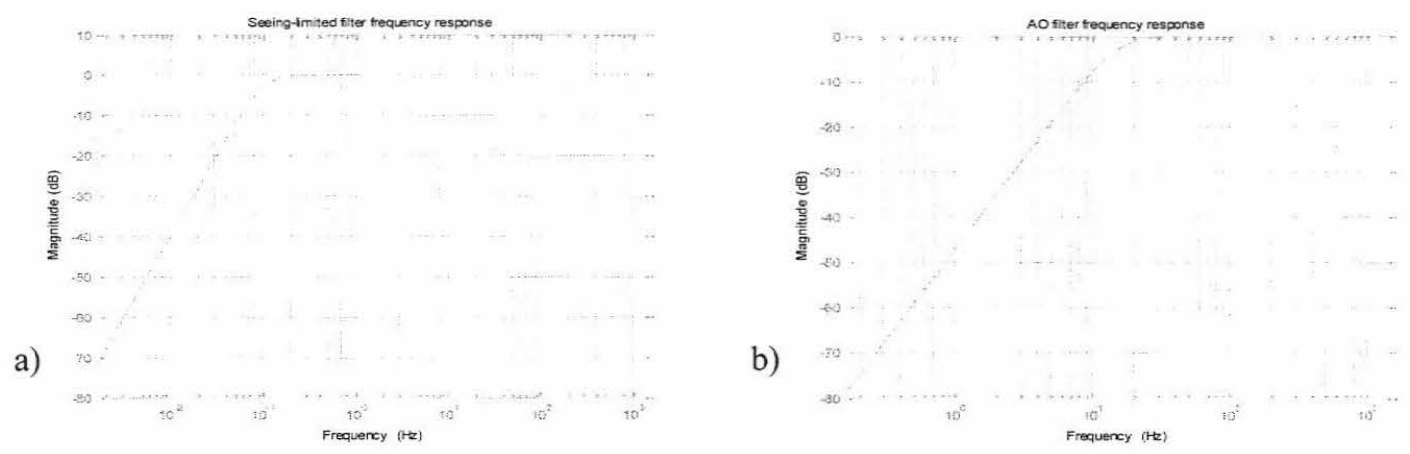

Figure 5. Bode plots of high-pass Butterworth filters representing a) the guided-tracking image jitter correction and b) AO image jitter correction.

Adaptive optic system image jitter (AO jitter) is corrected within the NFIRAOS instrument. The image location of a natural guide star is monitored on the wavefront detector and correction commands are sent to a fast steering tip/tilt mirror. Some of the natural guide stars are faint enough that the wavefront detector requires at least 67 milliseconds to collect enough energy to provide a strong enough signal for position control. Because of this, AO jitter errors can be can be corrected if they occur at periods greater than 67 milliseconds or with a frequency less than $15 \mathrm{~Hz}$. The AO jitter correction filter can be represented as a high pass Butterworth filter with a $15 \mathrm{~Hz}$ break frequency as shown in Figure $5 \mathrm{~b}$.

\section{TMT jitter allocations to telescope subsystems}

The image size error budget after guided-tracking operations is given in terms of PSS $_{\mathrm{N}}$ which is further described in ${ }^{[1]}$. After AO correction, the image size error budget is given in terms of nanometers RMS (nm $m_{R M S}$ ) of wavefront error. In Table 1 below, the error allocations for guided-tracking and AO image size are provided and also are converted to image motion on the sky in units of milliarcsec RMS (masRMS). The allocations for the secondary and tertiary mirror systems are divided into portions that represents motion of the mirror positioners and reaction motion of the telescope structure to positioner motions.

Table 1. Image Jitter Control Noise Error Allocations

\begin{tabular}{|c|c|c|c|c|c|c|c|}
\hline \multirow{3}{*}{$\begin{array}{l}\text { Requirement \# } \\
\text { [REQ-1-OAD-0454] }\end{array}$} & \multirow{3}{*}{$\begin{array}{c}\text { Description } \\
\text { Image jitter (control noise) }\end{array}$} & \multicolumn{3}{|c|}{ Guided Tracking Error allocation } & \multicolumn{3}{|c|}{ AO Error allocation } \\
\hline & & \multicolumn{2}{|c|}{$\left(\mathrm{PSS}_{\mathrm{N}}\right)$} & \multirow{2}{*}{$\begin{array}{l}\text { On-sky } \\
\left(\text { mas }_{\mathrm{RMS}}\right)\end{array}$} & \multicolumn{2}{|c|}{$\left(\mathrm{nm}_{\mathrm{RMS}}\right)$} & \multirow{2}{*}{$\begin{array}{c}\begin{array}{c}\text { On-sky } \\
\left(\mathrm{mas}_{\mathrm{RMS}}\right)\end{array} \\
0.471\end{array}$} \\
\hline & & 0.9947 & & & 17.0 & & \\
\hline [REQ-1-OAD-0470] & Guider Noise & & 0.9990 & 10.5 & & 0.0 & \multirow{2}{*}{0.25} \\
\hline [REQ-1-OAD-0472] & Mount Control Noise & & 0.9980 & 14.8 & & 9.0 & \\
\hline \multirow[t]{3}{*}{ [REQ-1-OAD-0474] } & M2 jitter & & 0.9978 & 15.4 & & 5.4 & 0.15 \\
\hline & M2 positioner jitter & & .99999 & 15.4 & & 5.4 & 0.15 \\
\hline & Telescope structure reaction & & .99999999 & na & & na & na \\
\hline \multirow[t]{3}{*}{ [REQ-1-OAD-0476] } & M3 jitter & & 0.9999 & 3.3 & & 13.4 & 0.37 \\
\hline & M3 positioner jitter & & 0.99993 & 3.3 & & 13.4 & 0.37 \\
\hline & Telescope structure reaction & & 0.9999999 & na & & na & na \\
\hline
\end{tabular}

\subsection{Secondary mirror jitter allocations}

The coordinate system used to describe secondary mirror motions is shown in Figure 6. The motions that will cause image jitter are translations along the $\mathrm{X}$ and $\mathrm{Y}$ axes, rotations about those axes, $\mathrm{RX}$ and $\mathrm{RY}$. The conversion factors between the secondary mirror motions and on-sky angular motion at the image are: 165.8 micrometers translation at the secondary mirror per arcsecond on the sky; and 5.3 arcseconds rotation at the secondary mirror per arcsecond on the sky. 
The portion of the $\mathrm{CN}$ jitter related to the response of the telescope structure at the secondary mirror interface is allocated $25 \%$ of the full M2 on-sky CN jitter. The remain $\mathrm{g} 75 \%$ is allocated to the internal $\mathrm{CN}$ jitter caused by leg motion errors of the secondary mirror hexapod positioner

The sensitivity of the on-sky image to secondary mirror translations and rotations in terms of changes in hexapod leg lengths were calculated using the following steps

1. Leg length values were calculated for 6 hexapod starting cases within the hexapod required range of motion: nominal, extreme $+X$, extreme $+Y$, extreme $+Z$, extreme $R x$, and extreme Ry.

2. Using backwards kinematics, motions of the secondary mirror in X, Y, Z, Rx and Ry from the starting cases were correlated to changes in leg lengths. By dividing mirror motions by the amount of leg length change, the sensitivity of X, Y, Z, Rx and Ry motions were determined per $\mu \mathrm{m}$ of leg length change.

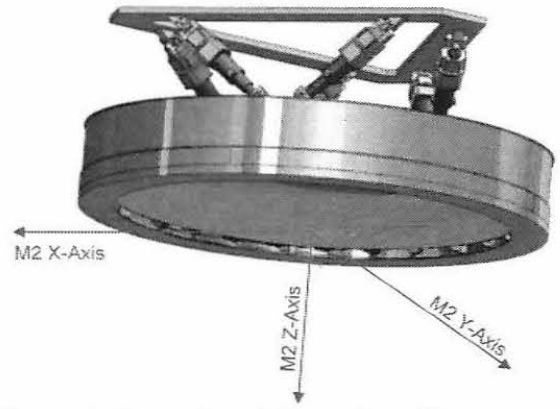

Figure 6. Secondary Mirror Coordinate system

3. Using the conversion factors, on-sky motions were calculated from X, Y, Rx and Ry per $\mu \mathrm{m}$ of leg length change to derive milliarcseconds (mas) on-sky/ $\mu \mathrm{m}$ leg motion.

4. Since $X$ and Ry motions cause on-sky motion along the telescope $X$ direction, and $Y$ and $R x$ motions cause onsky motion along the telescope $\mathrm{Y}$ direction, on-sky motions were summed to create $\mathrm{X}$ and $\mathrm{Y}$ image motions onsky. The $\mathrm{X}$ and $\mathrm{Y}$ were combined in quadrature to find the radius of on-sky error caused by $1 \mu \mathrm{m}$ of leg motion for each leg. The radii of on-sky error of all six legs moving $1 \mu \mathrm{m}$ were then combined in quadrature to derive the RSS mas on-sky per $1 \mu \mathrm{m}$ leg motion.

5. To determine the limit of hexapod leg motion that would create on-sky motion less than the jitter allocations, the combined on-sky radius of error caused by all six leg contributions per $1 \mu \mathrm{m}$ of leg motion is divided by the allocated on-sky error for guided tracking and $\mathrm{AO}$ jitter. The Table 2 summarizes the results.

Table 2. Allocation for Secondary Mirror Hexapod leg length jitter - these apply after filtering

\begin{tabular}{|c|c|c|}
\hline Required M2 allocation: & allocation & hexapod leg length error allocation \\
\hline guided tracking: & \begin{tabular}{l|l|}
15.4 & mas RMS
\end{tabular} & $<515 \mid \mathrm{nm}$ RMS \\
\hline AO corrected: & \begin{tabular}{l|l|}
0.15 & mas RMS \\
\end{tabular} & \begin{tabular}{l|l}
$<5.0$ & $\mathrm{~nm}$ RMS \\
\end{tabular} \\
\hline
\end{tabular}

\subsection{Tertiary mirror allocations}

The coordinate system used to describe tertiary mirror jitter motions, tilt $(\varphi)$, rotation $(\theta)$ and M3CRS Z-translation (ZM3), is shown in Figure 7. The tertiary mirror on-sky jitter allocations from Table 1 are shared by these three motions that effect on-sky image motion: rotation, $\theta\left(\mathrm{R}_{\theta}\right)$; tilt, $\phi\left(\mathrm{R}_{\phi}\right)$;, and translation in M3CRS-Z). Conversion factors from tertiary mirror motions to on-sky motions are given in Table 3.

Table 3. Conversion values: tertiary mirror motions to on-sky image motions

\begin{tabular}{|c|c|c|}
\hline \multicolumn{3}{|c|}{ Conversion Values } \\
\hline $\mathbf{X}_{\text {On-sky }} / \mathbf{R}_{\theta}$ & $\mathbf{Y}_{\text {On-sky }} / \mathbf{R}_{\varphi}$ & $\mathbf{X}_{\text {On-sky }} / \mathbf{Z}_{\mathrm{M} 3}$ \\
\hline $0.044 \operatorname{arcsec} / \operatorname{arcsec}$ & $0.089 \operatorname{arcsec} / \operatorname{arcsec}$ & $0.00068 \mathrm{arcsec} / \mu \mathrm{mn}$ \\
\hline
\end{tabular}

The tertiary mirror on-sky jitter allocations from Table 1 were divided into portions for each error motion that sum together in quadrature to equal the total allocation. The following assumptions were made in order to estimate the jitter allocation at the tertiary mirror for each motion:

- The rotation bearing radius $(\mathrm{Rr})$ is 1 meter and the tilt bearing radius ( $\mathrm{Rt}$ ) is 0.2 meters - a factor of 5 difference

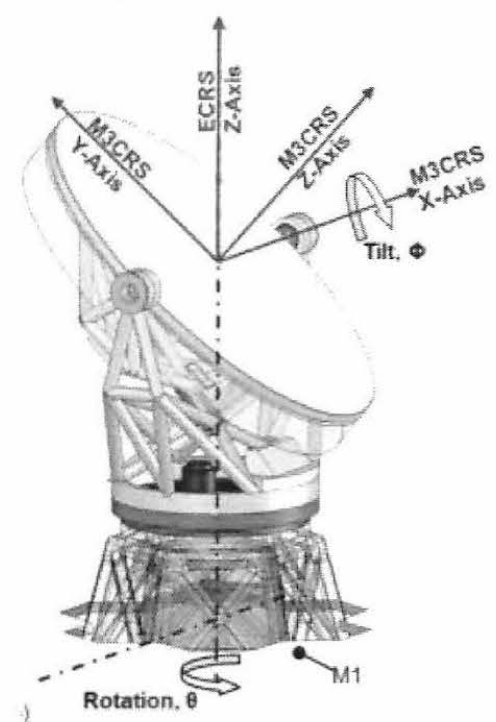

Figure 7. Tertiary Mirror Coordinate Systems 
- $\quad$ The spacing (St) between the tilt bearings is 3 meters

- $\quad \mathrm{Z}_{\mathrm{M} 3}=$ linear conversion of the radial jitter from the tilt bearing + linear conversion of the axial jitter from the rotation bearing

- Axial and radial jitter of the rotation and tilt drive systems are equal to their angular jitter

- Angular jitter is proportional to the radius of the bearing

With these assumptions, the following equations describe the jitter relationships at the tertiary mirror system:

$$
\begin{array}{ll}
R_{\theta} \text { jitter }=J & \text { (units: mas) } \\
R_{\varphi} \text { jitter }=\frac{J}{5} & \text { (units: mas) } \\
Z_{M 3} \text { jitter }=\left(\frac{R r}{\sqrt{2}} \times R_{\theta} j \text { itter }+\frac{S t}{2 \sqrt{2}} \times R_{\varphi} j \text { itter }\right) * 0.0048 & \text { (units: } \mu \mathrm{m} \text { ) }
\end{array}
$$

Using the conversion factors in

Table 3, the tertiary mirror local motions were converted to on-sky motions in terms of J, summed in quadrature, and set to equal the total allocation. The value for $\mathrm{J}$ was then calculated. From J, the allocations for each tertiary mirror motion were calculated and are summarized in Table 4.

Table 4. Tertiary positioner jitter allocations - these apply after filtering

\begin{tabular}{|c|c|c|}
\hline Tertiary positioner motions & $\begin{array}{c}\text { Allocated jitter } \\
\text { Guided Tracking }\end{array}$ & $\begin{array}{c}\text { Allocated jitter } \\
\text { AO Corrected }\end{array}$ \\
\hline $\mathrm{R}_{\theta}$ & $<69$ mas RMS & $<7.7$ mas RMS \\
\hline $\mathrm{R}_{\phi}$ & $<13.8$ mas RMS & $<1.5$ mas RMS \\
\hline $\mathrm{Z}_{\mathrm{M} 3}$ & $<0.34 \mu \mathrm{m}$ RMS & $<0.037 \mu \mathrm{m}$ RMS \\
\hline
\end{tabular}

\section{Options to manage the jitter requirement on the Secondary Tertiary mirror systems}

Because the jitter allocations for AO Corrected jitter are very small, several options are considered by TMT.

\subsection{Stepping option}

A method of reducing jitter is to pause mirrors during observing allowing the astronomical image to drift across the detector field of view. When the image reaches the edge of the field of view, the positioners would step the mirrors to a new location to place the image at the forward edge of the field of view. The positioners would then pause until the image drifted to the back edge of the field of view, then repeat. Observing time would be lost during step and settle repositioning time. Continuous observing will preserve observing time, increasing the telescope efficiency and is the optimal mode of operation. Because of this, this option is not preferred.

\subsection{Active cancellation option}

Image stabilization equipment could be added to the secondary and tertiary mirror subsystems that would sense jitter motions and command opposite compensating motions. This equipment is expensive and would increase the complexity of the secondary and tertiary mirror systems. Because of this, this option is not preferred.

\subsection{Passive option}

Careful component selection and hardware design may produce hardware that meets the stringent jitter requirements without additional cancellation. In order to understand the feasibility of this option, TMT has investigated performance of existing astronomical hardware to determine if similar existing systems meet the TMT jitter requirements.

Secondary mirror investigations: During a study effort in 2012, TMT was unable to find any hexapod system that had been tested with results meeting the TMT AO jitter requirements. In 2013, EELT engineers shared information with TMT about jitter testing at A.D.S. International, S.r.l (ADS) that was performed on an available hexapod arm actuator. 
ADS performed additional testing for TMT using data rates, velocities and motions that meet TMT requirements. The test details are described in Section 4 below.

Tertiary mirror investigations: During the study effort in 2012, several companies provided jitter performance data of existing systems similar to the tertiary mirror positioner that appeared to meet the TMT jitter requirements. In 2013, Advanced Mechanical \& Optical Systems S.A. (AMOS) offered to run tests for TMT on a 2 meter class telescope with a similar positioner that was available in their laboratory. AMOS performed tests on this existing system using TMT specified data rate, velocities, and motion profiles. The details of this test are shown in Section 5 below.

\section{TESTING TO INVESTIGATE SECONDARY MIRROR SYSTEM JITTER}

\subsection{Description of the hardware that was tested}

a)

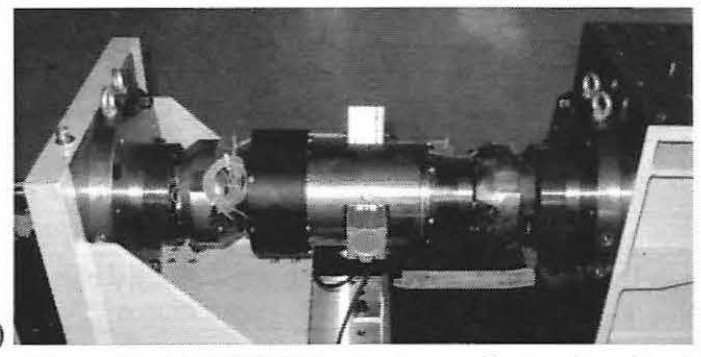

Figure 8: a) VLT-DSM actuator on the test bench b)

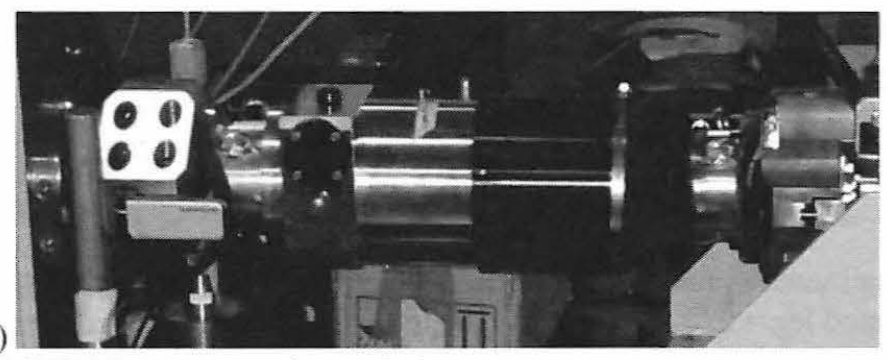

b) ADS FNAL actuator on the test bed

ADS provided raw data from testing performed on an ESO VLT-DSM hexapod spare actuator shown in Figure 8a. The tested hexapod actuator differs from the possible TMT hexapod in that it was designed for a much smaller payload (10x less), and uses a direct drive rather than worm-wheel gearing. Both the VLT and the TMT hexapod systems use a roller screw and closed-loop position control with a rotary encoder mounted to the roller screw which makes the jitter behavior credibly comparable between the two systems.

Figure $8 \mathrm{~b}$ shows an ADS FNAL hexapod actuator that is similar to what the TMT secondary mirror will require for its hexapod. The FNAL actuator has a 1:60 worm-wheel gearbox and an applied load that is the same order of magnitude as the TMT required load of 6 metric tons. The FNAL actuator is a leg of a hexapod system that supports the Dark Energy Camera (DEC) of the Dark Energy Survey. The DEC hexapod supports a load up to 3.5 metric tons and has been tested for survival at loads up to 8 metric tonnes. Figure 9 shows additional comparison between the two actuators.

a)

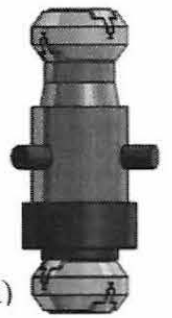

b)

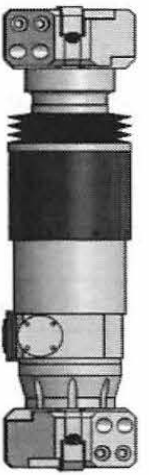

Figure 9. Comparison between a) VLT-DSM actuator (total length $498 \mathrm{~mm}$ ) and b) FNAL actuator (total length $782 \mathrm{~mm}$ ).

\subsection{Description of the test set-up}

The test was performed on a bench set-up using internal and external encoders to measure position. Measured position was compared to commanded position to calculate following error. The test data was collected at a high frequency rate, $2250 \mathrm{~Hz}$. The rotary encoder mounted aboard the actuator is the Heidenhain EQN 425 with a resolution of $0.24 \mathrm{~nm} / \mathrm{bit}$ of actuator linear displacement. The external linear encoder that was used to measure the actuator external motion is a linear encoder Heidenhain LF183, with a resolution 0.98 $\mathrm{nm} / \mathrm{bit}$. Data from the linear external encoder provides comparison to data from the internal rotary encoder. Additionally, typical test conditions in the ADS laboratory environment results in RMS noise of $1.9 \mathrm{~nm}$ in the external encoder and $0.3 \mathrm{~nm}$ in the internal rotary encoder. Figure 10 shows the test set-up.

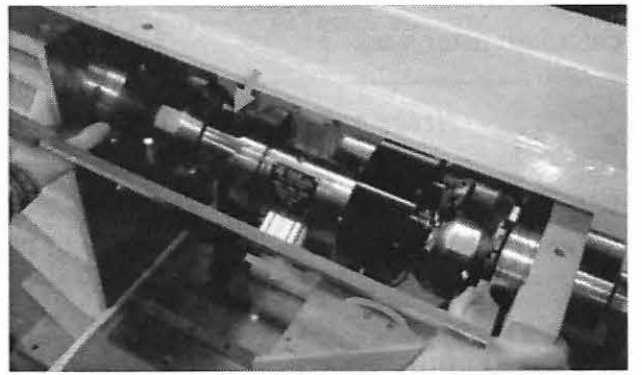

Figure 10. VLT-DSM actuator on the test bench. As a reference, the operator measures the actuator length via hand caliper. Red arrow shows the LF 183 linear external encoder (fixed part mounted on the test bench, sliding part installed below the mobile stage, circled in red); blue arrow indicates the position of the internal rotary encoder 
A difference between the test that was performed on the VLT-DSM actuator and the operational conditions of the TMT secondary mirror hexapod is that the tested hexapod leg actuator was not loaded during the test. The TMT secondary mirror hexapod will always be loaded in tension during observing. The ADS test set is instrumented to provide compression load using a hydraulic piston system. The pump that pressurizes the hydraulics creates vibration up to $100 \mathrm{~nm}$ magnitude. This load system is noisy and not appropriate for TMT so was turned off for this test.

\subsection{Hexapod actuator test plan}

The tests encompassed the range of velocities required by the hexapod leg actuators during telescope observing. For the secondary mirror system, the required maximum velocities are shown in Table 5.

Table 5. TMT M2S Hexapod maximum velocity requirement

\begin{tabular}{|c|c|c|c|c|c|}
\hline \multirow{2}{*}{ M2 System Tracking Motion } & $\mathrm{x}$ & $\mathrm{y}$ & $\mathrm{z}$ & $\mathrm{Rx}$ & $\mathrm{Ry}$ \\
\hline \multirow{2}{*}{ Maximum Velocity } & 0.001 & 0.001 & 0.001 & 0.068 & 0.001 \\
\cline { 2 - 6 } & $\mathrm{mm} / \mathrm{sec}$ & $\mathrm{mm} / \mathrm{sec}$ & $\mathrm{mm} / \mathrm{sec}$ & $\operatorname{arcsec} / \mathrm{sec}$ & $\operatorname{arcsec} / \mathrm{sec}$ \\
\hline
\end{tabular}

Using these requirements, ADS performed a kinematic study to determine the resulting maximum linear velocity of a hexapod leg $=0.78 \mu \mathrm{m} / \mathrm{second}$. The tests that were performed are listed in Table 6 .

ADS collected position data at a sampling rate of $2250 \mathrm{~Hz}$ which characterizes the jitter frequency response well. The duration of each test was 4.9 seconds. The data collected included commanded position, and positions measured by the internal encoder and the external encoder. Jitter, also called following error, is the commanded position minus the measured position after zero-offset errors are removed.

Table 6. Hexapod actuator tests performed

\begin{tabular}{|c|c|c|l|}
\hline qty & Velocity & profile & Test ID numbers \\
\hline 1 & $10 \mu \mathrm{m} / \mathrm{sec}$ & Constant & TMT1000 \\
\hline 1 & $0 \mu \mathrm{m} / \mathrm{sec}$ & Constant & TMT1001 \\
\hline 1 & 0 to $0.8 \mu \mathrm{m} / \mathrm{sec}$ & From $0-0.8$ & TMT1002 \\
\hline 6 & $0.8 \mu \mathrm{m} / \mathrm{sec}$ & Constant & TMT1003, TMT1004, TMT1005, TMT1006, TMT1007, TMT1008 \\
\hline 3 & $0.08 \mu \mathrm{m} / \mathrm{sec}$ & Constant & TMT1009, TMT1010, TMT1011 \\
\hline
\end{tabular}

\subsection{Data analysis of the hexapod actuator jitter tests}

Each test provided data sets of the commanded and measured internal and external encoder readings. The equation used to calculate jitter was:

$$
\text { jitter }=\text { commanded position }-(\text { measured position }- \text { off set error })
$$

The unfiltered internal and external jitter was processed using Fourier analysis to produce periodograms of power spectral density versus angular frequency to understand whether there are frequencies that contribute strongly to jitter. The internally-measured and externally-measured jitters were then filtered using the second-order Butterworth high-pass filters with break frequency $0.1 \mathrm{~Hz}$ for guided tracking and $15 \mathrm{~Hz}$ for $\mathrm{AO}$ correction to create data sets of remaining jitter after each filtering process. The RMS was calculated for internal and external jitter data sets: unfiltered jitter, guided tracking filtered jitter, and AO correction filtered jitter. The RMS jitter results are presented in Table 7. 
Table 7. Summary hexapod actuator RMS jitter values unfiltered, tracking filtered, and AO filtered

\begin{tabular}{|c|c|c|c|c|c|c|c|}
\hline \multirow[t]{2}{*}{ Test ID } & \multirow{2}{*}{$\begin{array}{l}\text { Velocity } \\
(\mu \mathrm{m} / \mathrm{sec})\end{array}$} & \multicolumn{2}{|c|}{$\begin{array}{l}\text { Unfiltered RMS jitter } \\
\text { (nm RMS) }\end{array}$} & \multicolumn{2}{|c|}{$\begin{array}{l}\text { Tracking filtered RMS } \\
\text { jitter (nm RMS) }\end{array}$} & \multicolumn{2}{|c|}{$\begin{array}{c}\text { AO filtered RMS jitter } \\
\text { (nm RMS) }\end{array}$} \\
\hline & & Internal & External & Internal & External & Internal & External \\
\hline TMT1001 & 0.00 & 0.3 & 2.3 & 0.3 & 1.9 & 0.2 & 0.9 \\
\hline TMT1009 & 0.08 & 1.6 & 7.1 & 1.6 & 5.2 & 1.0 & 1.2 \\
\hline TMT1010 & 0.08 & 1.6 & 5.0 & 1.5 & 3.6 & 1.1 & 1.2 \\
\hline TMT1011 & 0.08 & 7.2 & 5.1 & 5.4 & 4.7 & 1.1 & 0.8 \\
\hline TMT1003 & 0.80 & 20.3 & 29.0 & 15.0 & 20.8 & 1.1 & 1.9 \\
\hline TMT1004 & 0.80 & 18.9 & 27.2 & 17.8 & 21.4 & 1.2 & 2.1 \\
\hline TMT1005 & 0.80 & 46.6 & 68.4 & 34.1 & 45.9 & 1.9 & 3.0 \\
\hline TMT1006 & 0.80 & 19.6 & 17.6 & 13.9 & 12.3 & 1.3 & 1.5 \\
\hline TMT1007 & 0.80 & 27.5 & 27.2 & 16.9 & 16.6 & 1.2 & 1.4 \\
\hline TMT1008 & 0.80 & 36.7 & 37.0 & 25.3 & 23.3 & 1.7 & 1.7 \\
\hline TMT1000 & 10.00 & 88.2 & 105.0 & 96.0 & 102.5 & 6.6 & 6.8 \\
\hline TMT1002 & 0 to 0.8 & 125.7 & 121.0 & 119.4 & 124.6 & 1.5 & 3.7 \\
\hline \multicolumn{2}{|c|}{$\begin{array}{c}\text { TMT M2 hex leg jitter } \\
\text { requirements }\end{array}$} & & & \multicolumn{2}{|c|}{$<515 \mathrm{~nm}$ RMS } & \multicolumn{2}{|c|}{$<5.0 \mathrm{~nm}$ RMS } \\
\hline
\end{tabular}

\subsection{Results and conclusions of the hexapod actuator jitter test}

To understand differences between internal and external encoder measurements, the unfiltered RMS jitter values from both encoders were compared in the graph shown in Figure 11. The data points for each test are shown with a trend line that follows the average value at each velocity. The external jitter is close in value to the internal jitter, with the average values being slightly higher. The RMS jitter for every test meets the hexapod leg guided tracking allocation with no filtering by a margin of 5 times, even when traveling with a velocity that is 10 times higher than the maximum required velocity during observing.

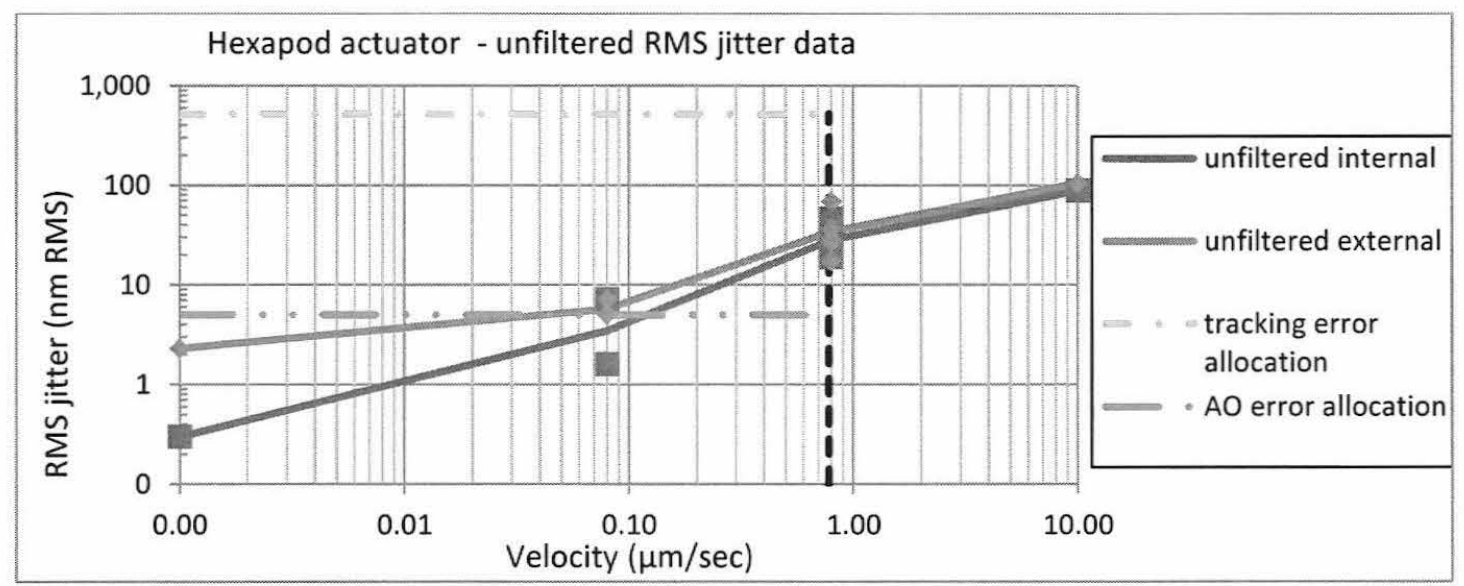

Figure 11. Unfiltered hexapod actuator RMS jitter vs angular velocity

A comparison of unfiltered and filtered RMS jitter data is shown in the graph in Figure 12Figure 12. Unfiltered and filtered hexapod actuator RMS jitter vs angular velocity. This graph shows that the AO filtered jitter RMS data meets the AO error allocation by a factor of 2.9 within the velocity ranges of the secondary mirror hexapod legs during observing. The guided tracking filtered data is included for completeness, and demonstrates that margin between the allocation and the filtered data has increased to a factor of 20 within the required angular velocity range. 


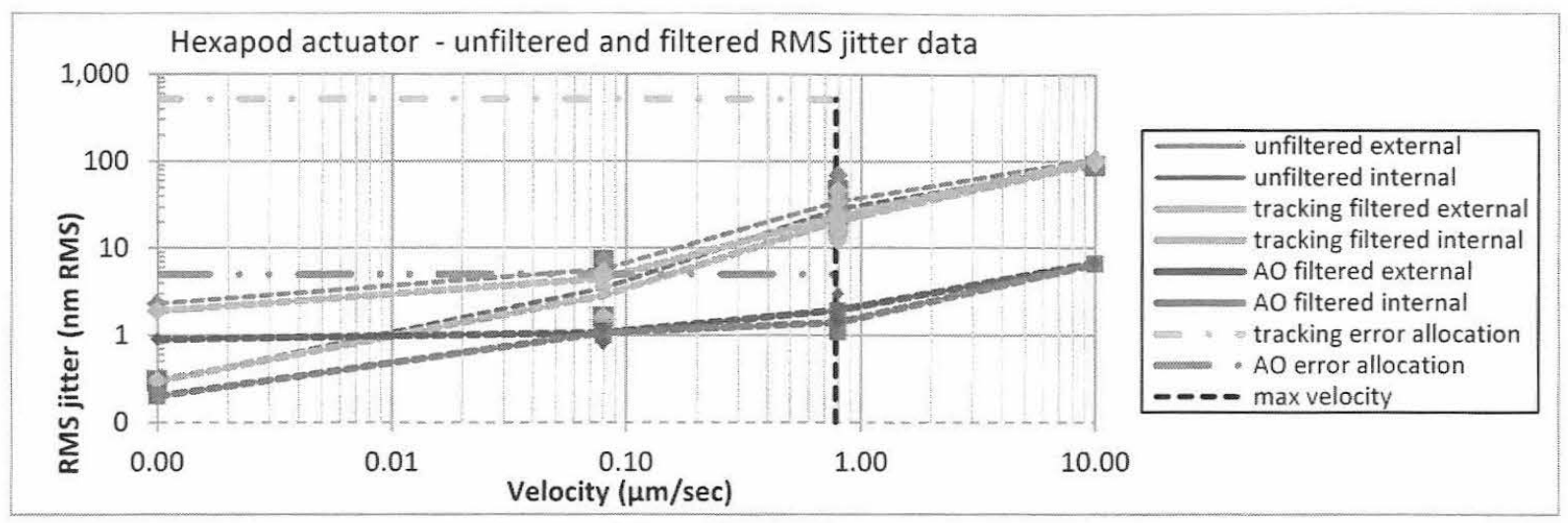

Figure 12. Unfiltered and filtered hexapod actuator RMS jitter vs angular velocity

The test TMT1002 was not included in the plots shown above since it spans multiple velocities from zero to $0.8 \mu \mathrm{m} / \mathrm{second}$. In this test, motion started from zero velocity, so stiction of the internal actuator bearings is represented. From Table 7, it can be seen that the internal and external jitter RMS of the unfiltered data from this test meets the secondary mirror hexapod leg jitter requirement for guided tracking filtered tracking by a margin of 4 , and that the $\mathrm{AO}$ corrected jitter meets the $\mathrm{AO}$ filtered allocation by a factor of 1.3 .

Figure 13 shows a sample periodogram of unprocessed jitter data collected at $10 \mu \mathrm{m} / \mathrm{second}$. This plot is representative of the periodogram of all tests and indicates that power response of the jitter is concentrated at the low frequencies.

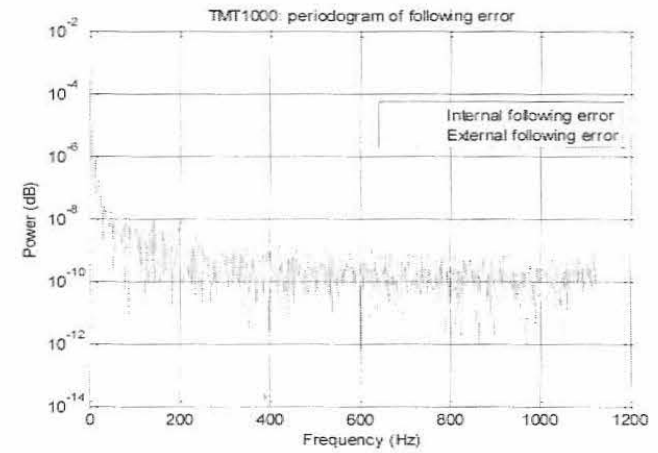

Figure 13. Periodogram of jitter collected at 10 micrometers/second showing power vs frequency

\section{TESTING TO INVESTIGATE TERTIARY MIRROR SYSTEM JITTER}

\subsection{Description of hardware that was tested}

The aim of the jitter testing was to measure performance of a configuration as similar as possible to the TMT tertiary mirror system. To achieve this, AMOS selected an altitude-azimuth telescope that was available at the AMOS factory in 2013. The telescope that was used was a 2 meter class so was similar in size to the TMT tertiary mirror system. Figure 14 shows a telescope that is similar to the one used for these tests.

\subsection{Description of the test set-up}

The mount control system of the tested telescope is based on a Delta Tau UMAC controller. Control of each axis is based on a PID loop with notch filters. To determine the frequency and depth of each notch filter, axis performance measurements were performed prior to the test. The control of the tested telescope was aimed at reducing the overall on-sky following error. No optimization was performed to influence the jitter frequency. Results could probably be improved if control were optimized to meet TMT tertiary mirror system requirements.

All testing was performed with a vertical azimuth axis so did not duplicate the gravity vector change that will be experienced by the TMT tertiary mirror system as the TMT telescope tilts in elevation.

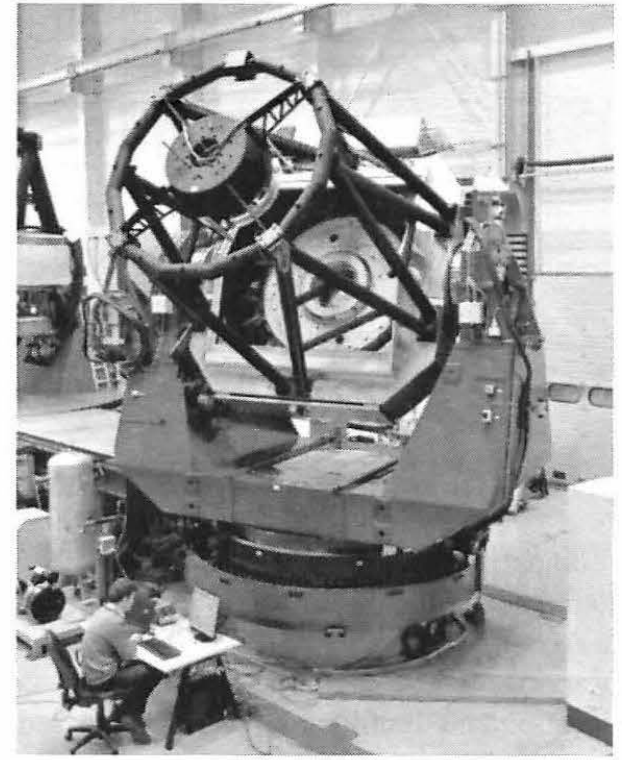

Figure 14. Pan-Starr 2 telescope - similar to the one tested. 


\subsection{Test plan}

The test plan was developed to encompass and go beyond the range of angular velocities required by the tertiary mirror positioner when the telescope is tracking an astronomical target. The maximum angular velocity for $R_{\theta}$ is $4.9 \operatorname{arcsec} / \mathrm{sec}$ and for $R_{\phi}$ is 3.3 arcsec/sec.

For each test identified with a constant velocity, the test profile included acceleration from stop to a constant angular velocity, maintained constant angular velocity for about 30 seconds, then decelerated back to stop. Angular velocities that were tested ranged from zero to $100 \mathrm{arcsecond} / \mathrm{second}$.

In addition, the telescope elevation angle increases then decreases as it tracks an astronomical object causing the tertiary mirror system rotations to smoothly slow through zero velocity while turning around to travel in the opposite direction. Since the motion instantaneously stops, bearing stiction is a concern; so this type of motion was included in the testing. Since these tests pass through zero angular velocity and the start and end velocities were less than $0.01 \mathrm{arcsec} / \mathrm{sec}$, these tests are identified as $<0.01$ velocity tests. The jitter from these tests was plotted at zero angular velocity. Additionally, tests were performed in both directions for each axis to determine whether a directional bias might exist.

AMOS provided data collected at a sampling rate of $200 \mathrm{~Hz}$ which characterizes the jitter frequency response well. The duration of each test was 32 seconds. The data collected included commanded position and position measured by an internal encoder. Jitter, also called following error, is the commanded position minus the measured position with any location offset errors removed. The tests that were considered for this analysis are listed in Table 8 .

Table 8 . Tests performed to study a 2 meter class telescope similar to the tertiary mirror system

\begin{tabular}{|c|c|c|c|c|c|}
\hline $\begin{array}{c}\text { Azimuth } \\
\text { Axis test } \\
\text { ID }\end{array}$ & $\begin{array}{c}\text { Altitude } \\
\text { Axis test } \\
\text { ID }\end{array}$ & $\begin{array}{c}\text { Velocity } \\
\text { arcsec/ } \\
\text { sec }\end{array}$ & Velocity profile & $\begin{array}{c}\text { Direction } \\
\text { of rotation }\end{array}$ \\
\hline T15 & T33 & $<0.01$ & Continuous decelerate to zero then accelerate & positive \\
\hline T16 & T34 & $<0.01$ & Accelerate from zero to constant velocity then decelerate & negative \\
\hline T17 & T35 & 0.01 & Accelerate from zero to constant velocity then decelerate & positive \\
\hline T18 & T36 & 0.02 & Accelerate from zero to constant velocity then decelerate & positive \\
\hline T19 & T37 & 0.05 & Accelerate from zero to constant velocity then decelerate & positive \\
\hline T20 & T38 & 0.10 & Accelerate from zero to constant velocity then decelerate & positive \\
\hline T21 & T39 & 1.00 & Accelerate from zero to constant velocity then decelerate & positive \\
\hline T22 & T40 & 5.00 & Accelerate from zero to constant velocity then decelerate & positive \\
\hline T23 & T41 & 10 & Accelerate from zero to constant velocity then decelerate & positive \\
\hline T24 & T42 & 100 & Accelerate from zero to constant velocity then decelerate & negative \\
\hline T25 & T43 & -0.01 & Accelerate from zero to constant velocity then decelerate & negative \\
\hline T26 & T44 & -0.02 & Accelerate from zero to constant velocity then decelerate & negative \\
\hline T27 & T45 & -0.05 & Accelerate from zero to constant velocity then decelerate & negative \\
\hline T28 & T46 & -0.10 & Accelerate from zero to constant velocity then decelerate & negative \\
\hline T29 & T47 & -1.00 & Accelerate from zero to constant velocity then decelerate & negative \\
\hline T30 & T48 & -5.00 & Accelerate from zero to constant velocity then decelerate & negative \\
\hline T31 & T49 & -10 & Accelerate from zero to constant velocity then decelerate & negative \\
\hline T32 & T50 & -100 & & & \\
\hline & & & &
\end{tabular}

\subsection{Data analysis}

For the tests with turn-around profiles, the entire time period measured was used since accelerations were less than the maximum TMT requirements for both axes. For tests that reached constant velocities, only the constant velocity portion of the data was processed since the accelerations were larger than TMT maximum observing accelerations. Jitter data was filtered using second order Butterworth high-pass filters with break frequency $0.1 \mathrm{~Hz}$ for guided tracking filtering and break frequency $15 \mathrm{~Hz}$ for $\mathrm{AO}$ correction filtering to create filtered jitter data sets. RMS jitter was calculated for each data set: raw jitter and two filtered jitter data sets. In addition, the raw and filtered jitter data was processed using Fourier analysis to produce periodograms of power spectral density versus frequency to understand whether there are strong jitter power responses at certain frequencies. RMS jitter results are listed in Table 9. 
Table 9. RMS jitter results from testing on $2 \mathrm{~m}$ class telescope

\begin{tabular}{|c|c|c|c|c|c|c|c|c|c|c|}
\hline $\begin{array}{c}\text { Angular } \\
\text { velocity } \\
\text { (arcsec/ sec) }\end{array}$ & $\frac{\infty}{x}$ & $\stackrel{\vec{\sigma}}{*}=$ & $\begin{array}{c}\text { raw jitter } \\
\text { (mas RMS) }\end{array}$ & $\begin{array}{c}\text { tracking } \\
\text { filter applied } \\
\text { (mas RMS) }\end{array}$ & $\begin{array}{c}\text { AO filter } \\
\text { applied (mas } \\
\text { RMS) }\end{array}$ & $\frac{n}{x}$ & $\stackrel{\overrightarrow{0}}{*} \ominus$ & $\begin{array}{l}\text { raw jitter } \\
\text { (mas RMS) }\end{array}$ & $\begin{array}{c}\text { tracking filter } \\
\text { applied } \\
\text { (mas RMS) }\end{array}$ & $\begin{array}{c}\text { AO filter } \\
\text { applied } \\
\text { (mas RMS) }\end{array}$ \\
\hline$<0.01$ & \multirow{18}{*}{ 产 } & T15 & 19.02 & 2.61 & 1.35 & \multirow{18}{*}{$\stackrel{\frac{0}{\Xi}}{\stackrel{\Xi}{E}}$} & T33 & 0.91 & 0.91 & 0.71 \\
\hline 0.01 & & T17 & 4.11 & 3.75 & 1.98 & & T35 & 0.99 & 0.99 & 0.77 \\
\hline 0.02 & & T18 & 3.74 & 3.49 & 1.87 & & T36 & 0.97 & 0.96 & 0.77 \\
\hline 0.05 & & T19 & 3.17 & 3.05 & 1.71 & & T37 & 1.00 & 0.99 & 0.78 \\
\hline 0.1 & & $\mathrm{~T} 20$ & 2.88 & 2.81 & 1.52 & & T38 & 0.98 & 0.98 & 0.77 \\
\hline 1 & & $\mathrm{~T} 21$ & 4.20 & 3.04 & 1.47 & & T39 & 1.00 & 1.00 & 0.75 \\
\hline 5 & & $\mathrm{~T} 22$ & 5.81 & 3.40 & 1.21 & & T40 & 0.94 & 0.94 & 0.75 \\
\hline 10 & & $\mathrm{~T} 23$ & 5.23 & 4.13 & 1.27 & & T41 & 0.99 & 0.98 & 0.78 \\
\hline 100 & & T24 & 18.62 & 18.47 & 10.61 & & T42 & 2.57 & 2.56 & 1.71 \\
\hline$<-0.01$ & & T16 & 19.60 & 2.88 & 1.41 & & T34 & 0.88 & 0.88 & 0.72 \\
\hline-0.01 & & T25 & 3.60 & 3.52 & 1.85 & & T43 & 0.92 & 0.91 & 0.74 \\
\hline-0.02 & & $\mathrm{~T} 26$ & 3.40 & 3.36 & 1.79 & & T44 & 0.90 & 0.90 & 0.74 \\
\hline-0.05 & & T27 & 3.20 & 3.09 & 2.34 & & T45 & 0.89 & 0.89 & 0.73 \\
\hline-0.1 & & T28 & 3.18 & 2.95 & 1.60 & & T46 & 0.88 & 0.88 & 0.72 \\
\hline-1 & & T29 & 5.13 & 2.61 & 1.19 & & T47 & 0.89 & 0.89 & 0.72 \\
\hline-5 & & $\mathrm{~T} 30$ & 11.98 & 5.05 & 1.27 & & T48 & 0.94 & 0.93 & 0.74 \\
\hline-10 & & T31 & 6.88 & 4.26 & 1.26 & & T49 & 0.87 & 0.86 & 0.71 \\
\hline-100 & & T32 & 15.44 & 15.34 & 8.87 & & T50 & 2.59 & 2.47 & 1.44 \\
\hline \multicolumn{4}{|c|}{ Azimuth Allocated jitter (mas RMS) } & 69 & 7.7 & \multicolumn{3}{|c|}{ Altitude allocated jitter } & 13.8 & 1.5 \\
\hline
\end{tabular}

\subsection{Results and conclusions of the $2 \mathrm{~m}$ class telescope jitter test}

To understand whether direction of rotation influenced the raw jitter response, the RMS jitter values from positive rotations were compared to those of negative rotations. RMS jitter values from tilt and rotation were plotted versus absolute angular velocity. The results are shown in Figure 15 and Figure 16.

These figures demonstrate that the jitter is independent of direction of motion. The azimuth axis raw RMS jitter meets the guided tracking requirement; but does require $\mathrm{AO}$ correction filtering to meet the $\mathrm{AO}$ corrected requirement. Also, the azimuth jitter RMS during the turn-around at zero angular velocity is high - indicating that stiction may be a factor. Figure 16 shows that for the altitude axis, the raw RMS jitter meets both the guided tracking and the AO corrected allocations without any filtering for all angular velocities that will occur during observing.

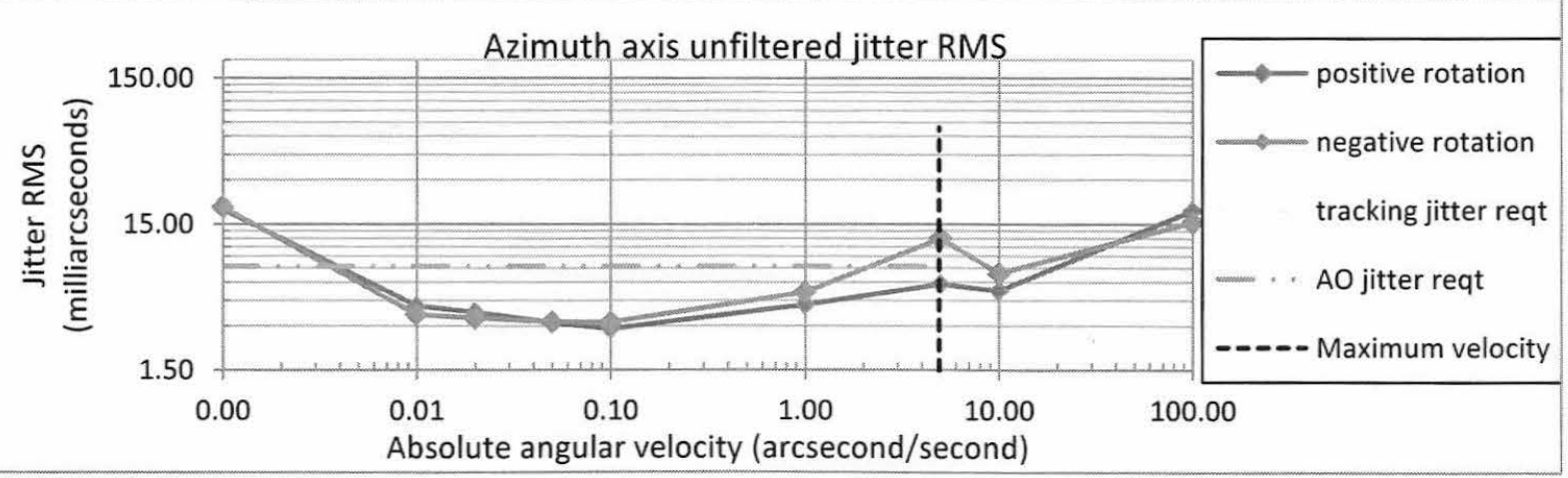

Figure 15. 2 meter class telescope azimuth axis raw jitter RMS vs absolute angular velocity for azimuth rotations 


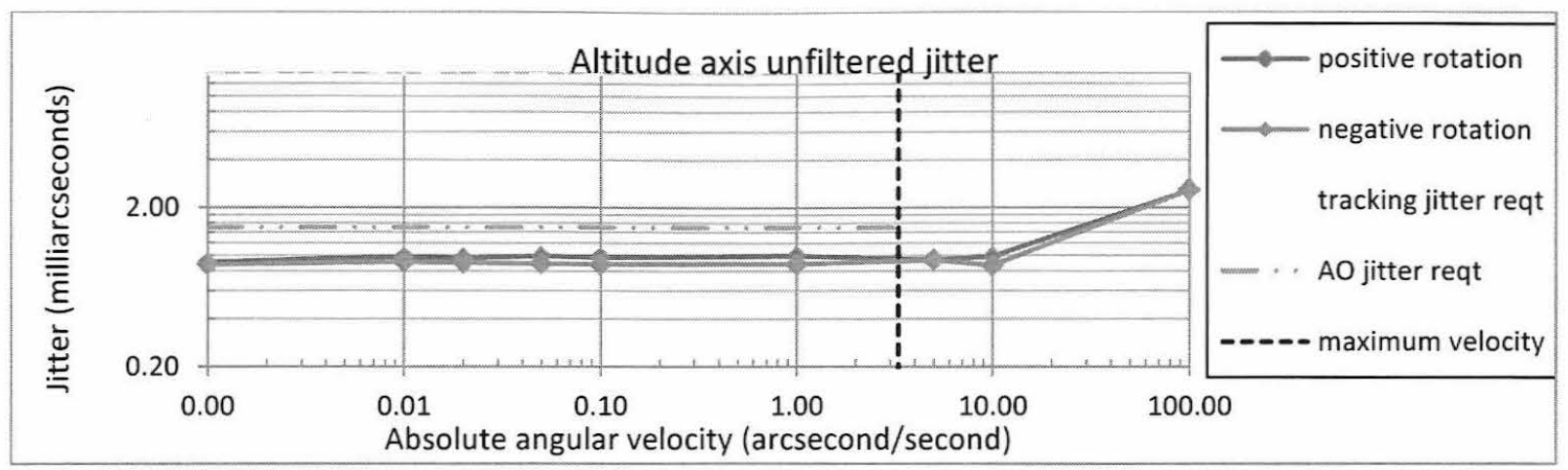

Figure 16. 2 meter class telescope altitude axis raw jitter RMS vs absolute angular velocity for altitude rotations

Because direction of motion did not significantly affect the raw jitter RMS values, absolute values of the angular velocities were considered in the remaining results. Figure 17 and Figure 18 show the effect on jitter RMS of guided tracking filtering and $\mathrm{AO}$ corrected filtering. In these plots, each data point is plotted, and a trend line follows the average jitter RMS for each velocity. For the azimuth data in Figure 17, filtered RMS jitter data meets both guided tracking and $\mathrm{AO}$ corrected requirements. Filtering reduces the zero angular velocity jitter RMS. For guided tracking filtering, the margin at the worst data point within the set of observing angular velocities is a factor of 13.7 times. For $\mathrm{AO}$ corrected filtering, the margin at the worst data point within observing angular velocities is a factor of 3.3 times.

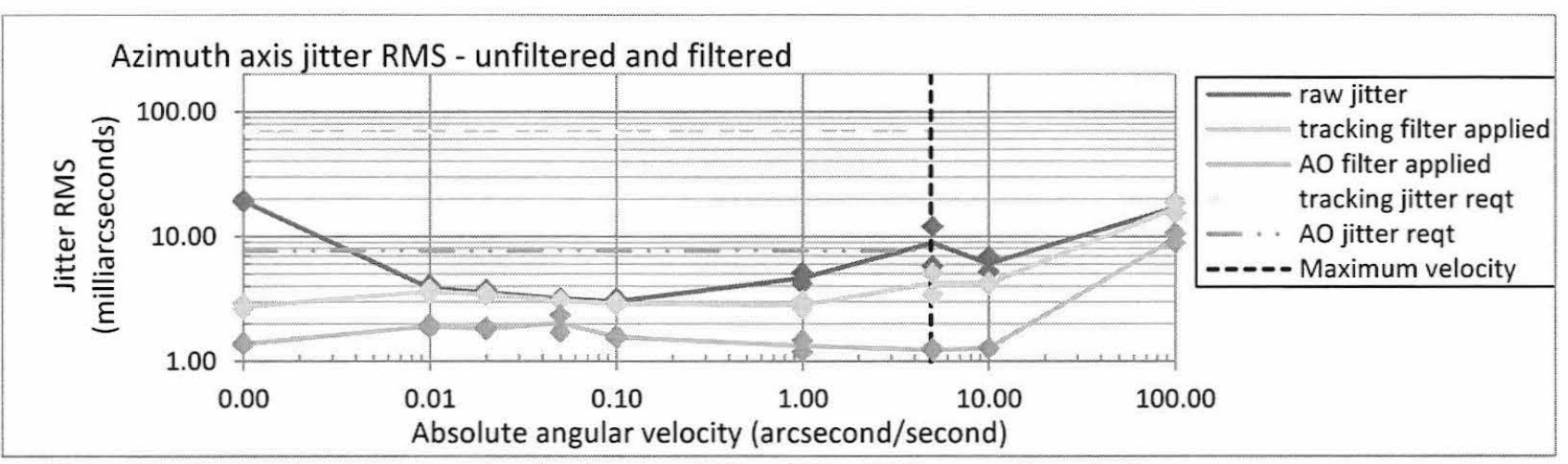

Figure 17. 2 meter class telescope azimuth axis raw and filtered jitter RMS vs angular velocity

The filtered altitude axis jitter RMS meets the requirements with margins of 13.8 times for guided tracking, and 2 times for $\mathrm{AO}$ corrected as demonstrated in Figure 18.

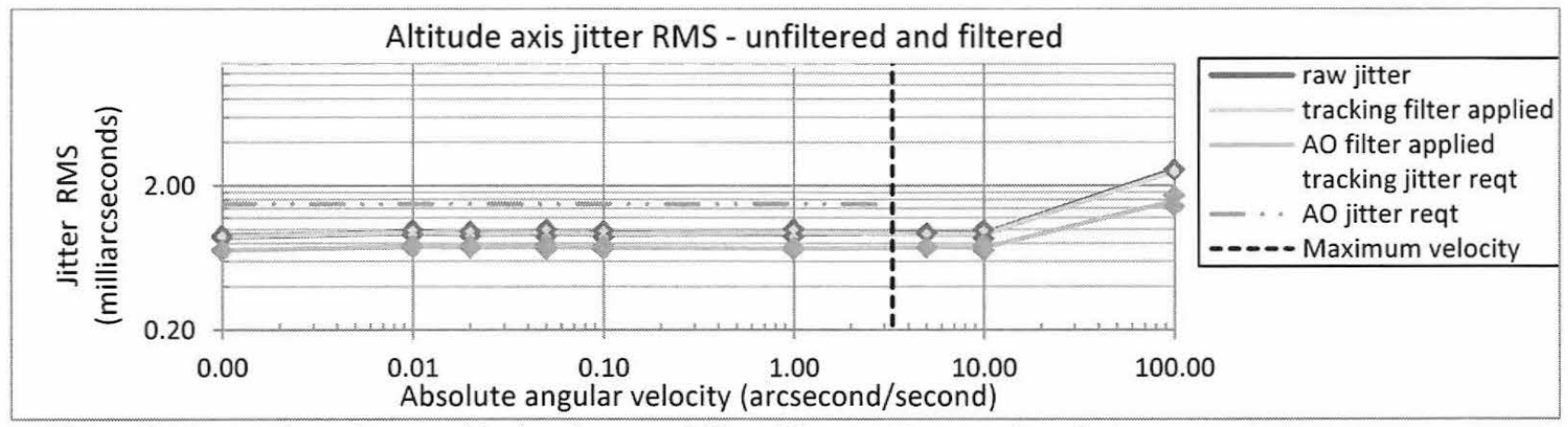

Figure 18. 2 meter class telescope altitude axis raw and filtered jitter RMS vs angular velocity

Using Equation (3) and average RMS jitter values of each axis at each angular velocity, values were calculated for zdirection raw and filtered jitter. The results are plotted in Figure 19 and indicate that the Z-direction jitter will meet allocations with margins of 18 times for guided tracking and 3.5 times for $\mathrm{AO}$ corrected. 


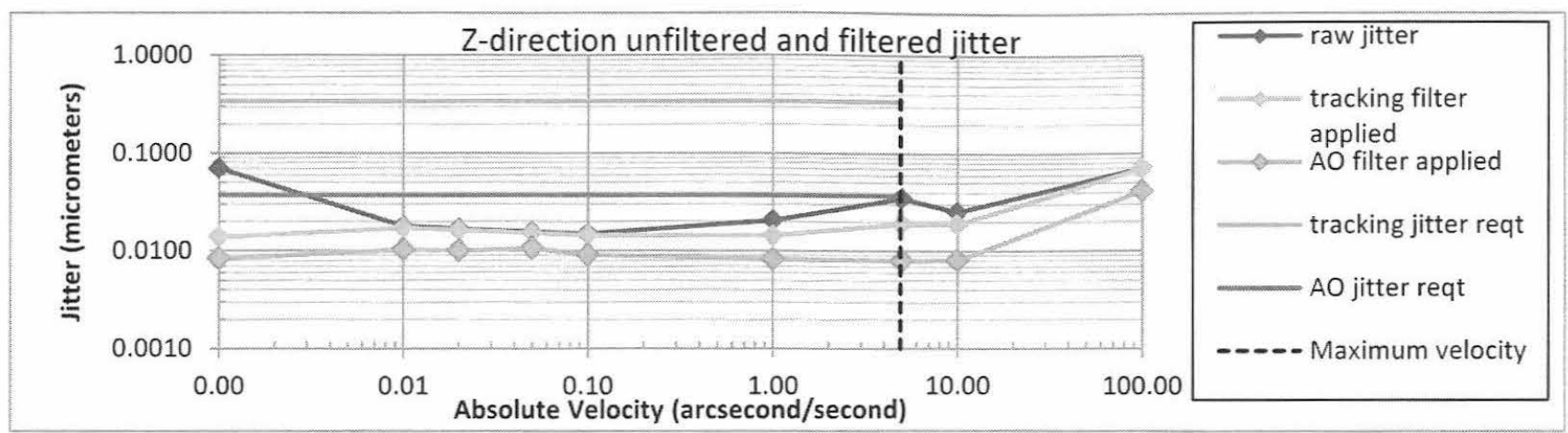

Figure 19. Z-direction jitter calculated from 2 meter class telescope data

To further understand the azimuth axis jitter performance during an angular velocity turn-around of $<0.01 \mathrm{arcsec} / \mathrm{sec}$ (plots a, b, and c) and at 5 arcsecond/sec (plots d, e, and f), periodograms of the jitter data sets are shown below in Figure 20. The raw jitter and the jitter after guided tracking have very similar frequency characteristics as shown in plots a, b, d, and e. The AO correction filters much of the jitter below $10 \mathrm{~Hz}$ as shown in charts $\mathrm{c}$ ) and $\mathrm{f}$ ). There is a strong jitter component at $10 \mathrm{~Hz}$ that is not filtered away and shows up in all charts. A response appears at $50 \mathrm{~Hz}$ that appears in 7 out of the 16 azimuth axis tests at a random selection of angular velocities.
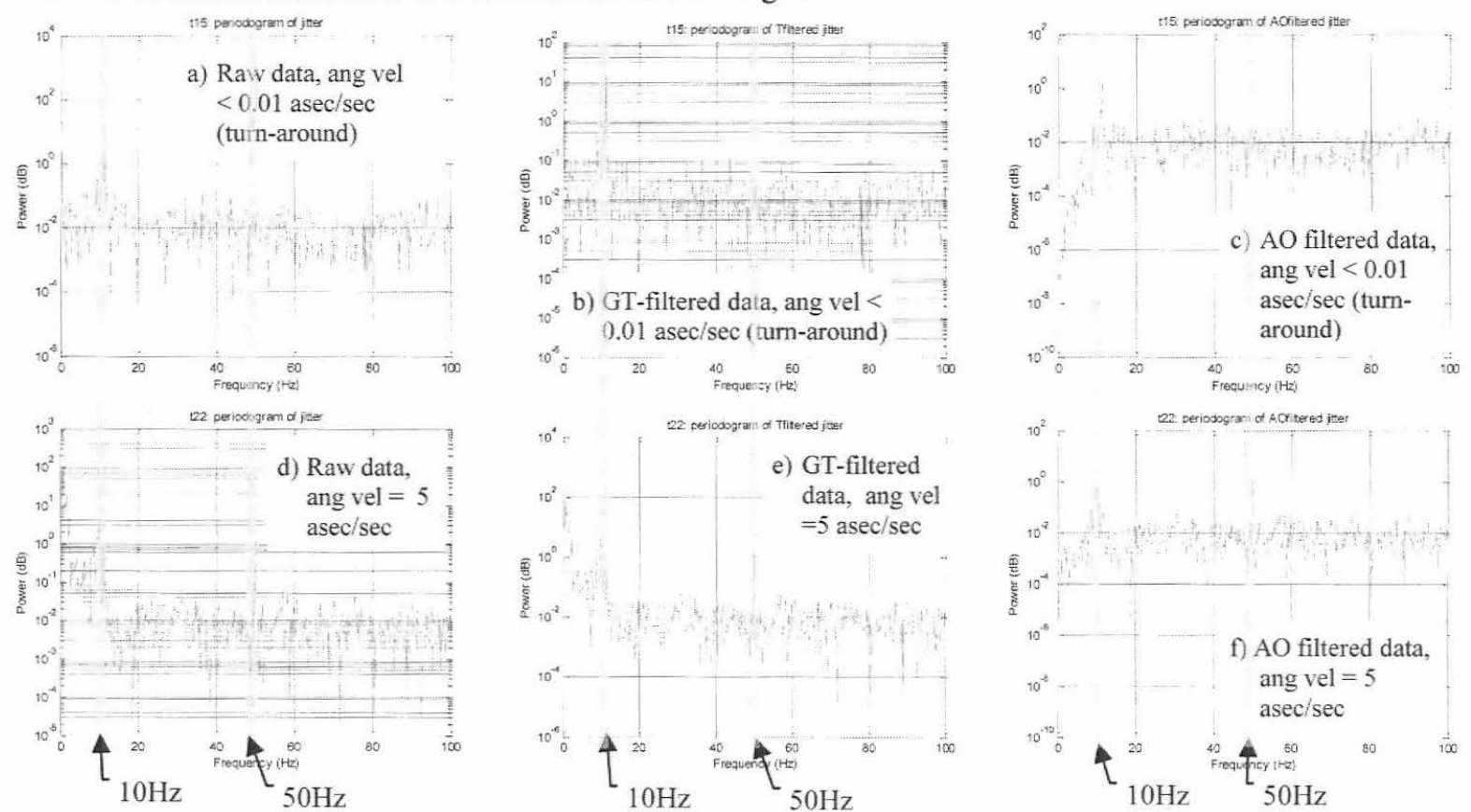

Figure 20. Jitter frequency response of two azimuth rotation tests: unfiltered (raw); guided tracking (GT), and AO corrected (AO)

\section{SUMMARY CONCLUSIONS}

\subsection{Secondary mirror system jitter test conclusion}

What was not included was turn-around motion where instantaneous zero velocity at the turn-around point may affect jitter. Because test TMT1002 started at zero velocity, it did capture bearing stiction effects, and the jitter from test TMT 1002 did meet requirements. It will be necessary to test turn-around motions to fully understand the resulting jitter when the motion is continuous, zero velocity is instantaneous, and direction of travel reverses. 
Other drawbacks of the test are that the hexapod actuator has differences from a hexapod actuator that could meet the secondary mirror requirements. Also, this test did not include performance of any possible jitter sources in the secondary mirror cell or from joint rotation in an entire hexapod system. Since the secondary mirror cell is passive, jitter sources would arise from structural motion in response to positioner jitter. Careful design considering structural response of the cell assembly will be required to meet requirements. Also, the effect of variable thermal environment and loading on the hexapod actuator has not been studied.

Since there is margin between jitter that was measured and the requirement for the secondary mirror, TMT will continue to proceed with the passive option. TMT plans include dedicated testing of the secondary mirror positioner during the construction phase between preliminary design phase and final design phase. These tests will cover shortfalls mentioned above with the exception of the cell design. That will be covered by the secondary mirror cell supplier.

The test results demonstrate that a hexapod actuator exists that can meet the jitter requirements for the TMT secondary mirror hexapod leg with significant margin. This was not known prior to this test and is a very welcome result to TMT.

\subsection{Tertiary mirror system jitter test conclusion}

The test of the 2 meter class telescope jitter performance included many important features. It included an entire system that is very similar to the tertiary mirror, thus captured the jitter performance of many subsystems in the measured data; such as: motor ripple, cable wrap jitter, encoder errors, bearing jitter and structural resonant responses. One shortfall of the test was that jitter response was collected at the internal encoders so structural interaction with the mirror cell has not been measured. Another shortfall is that the tertiary mirror system on TMT rotates with the telescope structure in elevation, so there will be significant moment loads on the bearings. Since the 2 meter class telescope is mounted on a stationary base, its jitter performance does not include a varying gravity vector and the higher bearing preload that will be required. In addition, no thermal variation testing was performed.

The supplier of the tertiary mirror system, Changchun Institute of Optics, Fine Mechanics and Physics, is currently building a $1 / 4$ scale prototype of the tertiary mirror system and a full scale prototype of the rotation drive system. Several sets of tests are planned on both prototypes to understand the jitter performance and to learn what is required to meet the TMT requirements.

In the meantime, the results from the testing that was done demonstrate that a system that is similar to the TMT tertiary mirror meets TMT requirements with significant margins when operated with velocities and motion profiles that duplicate those required by the tertiary mirror system. This is a very good result for TMT. Studies had reported that industry had built hardware that met requirements, but test profiles were not tailored to meet TMT requirements. These results using tailored angular velocities are very welcome to TMT.

\section{REFERENCES}

[1] B.J.SEO et al, "Analysis of Normalized Point Source Sensitivity as a performance metric for the Thirty Meter Telescope," Proc. SPIE 7017, (2008)

\section{ACKNOWLEDGEMENTS}

I would like to acknowledge the expertise, guidance and support provided by management staff of ADS International, S.r.1, and Advanced Mechanical \& Optical Systems S.A.

The TMT Project gratefully acknowledges support of TMT collaborating institutions. They are: Association of Canadian Universities for Research in Astronomy (ACURA), the California Institute of Technology, the University of California, the National Astronomical Observatory of Japan, the National Astronomical Observatories of China and consortium partners, and the Department of Science and Technology of India and supported institutes. This work was supported as well by the Gordon and Betty Moore Foundation, the Canada Foundation for Innovation, the Ontario Ministry of Research and Innovation, the National Research Council of Canada, the Natural Sciences and Engineering Research Council of Canada, the British Columbia Knowledge Development Fund, the Association of Universities for Research in Astronomy (AURA), the U.S. National Science Foundation, and the National Institutes of Natural Sciences of Japan. 